The majority (73.0\%) of patients had a known psychiatric diagnosis, with the most frequent being depressive disorder (36.0\%) and emotionally unstable personality disorder (15.0\%). Almost half (48.0\%) had a history of self-harm, and $40.0 \%$ were under the influence of alcohol/illicit substances upon presentation to ED. The most common psychosocial triggers were conflict with partner (26.0\%) and stress due to the COVID-19 pandemic (19.0\%).

Following assessment, $24.0 \%$ of patients were discharged to their General Practitioner, $18.0 \%$ to the community mental health team; and $17.0 \%$ to the Crisis \& Home Treatment Team. A minority (13.0\%) were admitted to a psychiatric hospital (10.0\% informally, 3.0\% under the Mental Health Act 1983).

Approximately one in five (21.0\%) patients re-attended to MHLS within 3 months. Out of 37 patients that had previously been assessed by MHLS in the preceding 12 months; $37.8 \%$ were reassessed within 3 months $(\mathrm{p}<0.01)$.

Conclusion. In the studied sample, most (90\%) of psychiatric patients attended ED for self-harm or suicide, and a significant proportion had repeat attendance. Socioeconomic deprivation, substance misuse, relationship difficulties and stress due to the COVID-19 pandemic were major issues, alongside diagnosed depression and personality disorder. Focussed support for these issues may decrease ED attendance due to self-harm/suicidality.

\section{A description of the use of zuclopenthixol decanoate long-acting injection in a large mental health trust \\ Shay-Anne Pantall ${ }^{1 *}$, Emily Whitehouse ${ }^{2}$ and Lisa Brownell ${ }^{1}$ \\ ${ }^{1}$ Birmingham and Solihull Mental Health NHS Foundation Trust and ${ }^{2}$ University of Birmingham, Medical School \\ ${ }^{\star}$ Corresponding author.}

doi: 10.1192/bjo.2021.891

Aims. Adherence with antipsychotic medication is an important factor in the prevention of relapse in psychotic disorders such as schizophrenia. Long acting antipsychotic injections promote improved adherence. In recent years, second generation antipsychotic long-acting injections have become increasingly popular, and little has been written about the use of the older depot medications. Here, we explore the current use of one of the firstgeneration antipsychotic long acting injections in Birmingham and Solihull Mental Health NHS Foundation Trust.

Method. An 18-month retrospective case-note review of all patients who started zuclopenthixol decanoate during the first 6 months of $2018(n=45)$

Result. Key findings included: -

\section{- $71 \%$ were male}

- The mean age was 37 (range 19-65)

- The most common diagnoses were: schizophrenia (51\%), bipolar affective disorder (18\%) and schizoaffective disorder (13\%). We noted that 2 individuals $(4 \%)$ had a primary diagnosis of recurrent depressive disorder, $2(4 \%)$ had a primary diagnosis of emotionally unstable personality disorder.

- $60 \%$ of those who were prescribed zuclopenthixol decanoate discontinued it within the 18-month follow-up period.

- The vast majority of discontinuation occurred within the first 6 months, and after this, few individuals stopped treatment.

- The most common reason for discontinuation was side effects (57\%), with other reasons including patient choice (7\%), noncompliance $(7 \%)$, pregnancy $(4 \%)$, or needle phobia $(4 \%)$.

Conclusion. Zuclopenthixol decanoate has been used for individuals with both schizophrenia and paranoid psychosis (where it is licenced) and also occasionally for other indications. A high proportion discontinued the zuclopenthixol within 6 months, this generally being attributed to adverse effects. Those who were still receiving this medication at 6 months were very likely to continue to take it throughout the 18 months. We would therefore recommend robust monitoring for and management of adverse effects in the early phases of treatment.

The clinical complexity of cases of schizophrenia in a community mental health team: a 3 year comparison

Shay-Anne Pantall ${ }^{1 \star}$, Laxsan Karunanithy ${ }^{2}$, Hayley Boden ${ }^{2}$ and Lisa Brownell ${ }^{1}$

${ }^{1}$ Birmingham and Solihull Mental Health NHS Foundation Trust and ${ }^{2}$ University of Birmingham, Medical School

${ }^{*}$ Corresponding author.

doi: 10.1192/bjo.2021.892

Aims. To describe the changes in complexity and management of individuals with schizophrenia in a community mental health team (CMHT) over a three year period.

Background. It is often believed that individuals receiving care from CMHTs are those with low levels of complexity and risk, and are relatively stable, with more complex individuals being managed by assertive outreach or other specialist teams. Here, we describe changes in the complexity, comorbidity, service-usage and management, of patients with a diagnosis of schizophrenia in a CMHT between 2016 and 2019 .

Method. Data were collected from an electronic patient record system (RiO) for all individuals with schizophrenia in a CMHT in Birmingham ( $\mathrm{n}=84$ in 2016, $\mathrm{n}=71$ in 2019), examining demographic variables, comorbidity, use of mental health services and current management.

Result. Key findings included: -

- $63 \%$ were managed through care programme approach (CPA) in 2016 , compared to only $31 \%$ in 2019 .

- $21 \%$ had required home treatment or inpatient care in the preceding 12 months in 2016; this had improved to 8.5\% in 2019.

- Significant levels of psychiatric comorbidity, including addictions with almost half of patients (46.5\%) having a known history of substance use in 2019 , compared to only $15.5 \%$ noted in 2016.

- Pharmacological management has remained broadly similar; in $201621 \%$ patients were taking a combination of 2 antipsychotics compared to only $10 \%$ in 2019 and $25 \%$ were taking clozapine in 2016 (21\% in 2019). 39\% were prescribed a long acting antipsychotic injection in 2016, compared to $32 \%$ in 2019.

- In 2016, medication was being prescribed in the majority of cases within secondary care (55\%) patients and in primary care in only $21 \%$. GPs have now taken on greater prescribing responsibility in 2019 , prescribing in $44 \%$ of cases, with $47 \%$ being prescribed by the CMHT.

Conclusion. The acuity and management of individuals with a diagnosis of schizophrenia under the care of a CMHT has changed over a 3 year period. It is positive to note the reduced use of crisis services and lower rates of polypharmacy. There is a reduction in the proportion of patients receiving management through $\mathrm{CPA}$, and a move towards more medication being prescribed in primary care. The reasons for this change are however unclear and may reflect change in available resources, given that more than half of this group receive clozapine or long acting injections, and have high levels of comorbidity. 